\title{
CLASIFICACIÓN Y SEMIOLOGÍA DE LOS TRASTORNOS DEL LENGUAJE EN EL NIÑO
}

\author{
Mg. Abel Cuzcano Zapata \\ Docente de la Escuela Profesional de Psicología - $U M C H$
}

El desarrollo del lenguaje se encuentra estrechamente vinculado a la evolución psicológica general, por lo que su adquisición se halla inmersa dentro del mundo psíquico del individuo; no obstante, el desarrollo del lenguaje también está ligado a una evolución neuropsicológica progresiva, siguiendo procesos complejos que dependen del grado de maduración y de la fisiología del organismo, por una parte, y del ambiente sociocultural por otra.

Este complicado proceso cognitivo, y a la vez de naturaleza modular, se ve afectado por una serie de trastornos del lenguaje que involucran un amplio grupo de patologías con características y etiologías sumamente diversas, que pueden persistir en algunos casos, a lo largo de toda la vida; sin embargo, sus síntomas, manifestaciones, efectos y grado de severidad, dependerán de una compleja red de factores que interactúan a lo largo del tiempo. Por ello es importante resaltar que en el caso de los niños, los trastornos del lenguaje abarcan un amplio espectro de síntomas que pueden ir, desde dificultades ligeras e imperceptibles para el no especialista, hasta problemas muy severos y evidentes para cualquiera; y desde edades muy tempranas con retraso sólo en la etapa prelingüística y en la adquisición de las primeras palabras, hasta una afectación de las etapas posteriores.

\section{MODELOS EN LA ADQUISICIÓN DEL LENGUAJE}

Al ser el lenguaje un proceso tan complejo y tema con tantas posibilidades de análisis, existen numerosos enfoques y teorías que han planteado variadas visiones acerca de su adquisición, sin embargo, y sin afán de que sea la única alternativa esta podrían agruparse en tres orientaciones principales:

\section{I.I TEORÍAS INNATISTAS}

Entre los principales autores de esta orientación destacan Chomsky (1975), Lennerberg (1975) y Mc Neill (1970), quienes consideran que el acceso al lenguaje 
se produce gracias a una serie de habilidades innatas que posee el individuo. Dicho de otra manera, se asume que el niño, desde su nacimiento, está lingüísticamente programado para desarrollar el lenguaje.

Es así que Chomsky, citado por Greene (1999), señala que para adquirir las reglas gramaticales, el niño tiene que haber alcanzado una representación innata de los principios de la gramática universal. Complementariamente a lo señalado por Chomsky, Lennerberg (1975) manifiesta que el niño está biológicamente predispuesto para aprender el lenguaje a medida que su cerebro madura.

\section{I.2 TEORÍAS COGNITIVAS}

Según este punto de vista, la adquisición del lenguaje se efectúa progresivamente a lo largo de una serie de etapas, con todo debe resaltarse que el desarrollo cognoscitivo inicial se produce sin la presencia del lenguaje. Así, un bebé adquiere la información a través de sus experiencias, el juego, la imitación, la representación mental y conocimientos perceptivos y no verbales; sin embargo estas formas de adquirir información son limitadas, por lo que el lenguaje se convierte en el factor que proporciona las herramientas que posibilitan la separación del pensamiento y la acción, así como, la posibilidad de representar los objetos ausentes y los sucesos pasados, de allí que según esta perspectiva, el lenguaje llega a ser útil recién cuando funcionan las primeras estructuras mentales.

Inicialmente, durante la etapa senso motriz, el pensamiento está ligado a las acciones y a las cualidades sensoriales de los estímulos. Un bebé reacciona positivamente ante un tono de voz cariñoso y negativamente ante un tono irritado, sin tener ningún conocimiento de "lenguaje". Mac Namara citado por Le Normand (Narbona, 2000, p. 30) sugirió que los bebés reconocen también expresiones sociales, la gesticulación y los movimientos corporales del locutor, como ayuda para la comprensión.

De acuerdo a Piaget (1976), hasta que el pensamiento no es simbólico, el lenguaje no estimula el desarrollo cognoscitivo, de tal manera que sólo en ese momento se convierte en el medio natural para representar objetos ausentes y sucesos pasados. Al comienzo de la etapa preoperatoria y a través de las diferentes etapas del desarrollo, el lenguaje cumple muchas funciones (Fraca; Barrera, 1988), ayudando a los niños, entre otras cosas, a resolver nuevos problemas y a codificar situaciones pasadas similares, proporcionándoles nuevos esquemas alternativos.

Complementariamente Le Normand (Narbona, 2000, p. 30) indica que si se quiere conocer cuáles son los mecanismos subyacentes para la adquisición del lenguaje, 
lo primero que debe hacerse, es practicar un examen detallado del desarrollo de las estrategias; es decir, el análisis de la manera en la que el niño se apropia de su lengua, por medio de la definición de los principios operativos que adopta para poner en relación el significado de los enunciados y su forma, tanto en el contexto sociofamiliar, cómo en el sociocultural.

La misma autora plantea también que en esta concepción, lo primordial es "comprender cómo el pequeño llega a dominar las principales funciones lingüísticas (prosódicas, fonológicas, semánticas, morfosintácticas, pragmáticas o contextuales) sabiendo que, en las situaciones naturales de comunicación, estas diversas funciones entran en relaciones complejas de interacción, y que las lenguas naturales se caracterizan por un solapamiento parcial de las formas y funciones" (Narbona, 2000, p. 30).

\section{I.3 TEORÍAS INTERACCIONISTAS}

En esta perspectiva, el aprendizaje del lenguaje o cualquier otro, ocurre a medida que los niños interactúan y experimentan con su medio ambiente, formulando diversos esquemas sobre los cuales luego, construyen más esquemas.

De esta forma las personas aportan su grado de madurez, motivación, autodisciplina, carga biológica innata y predisposición o estilos de aprendizaje, a la actividad de construir esquemas a partir de sus interacciones con el medio ambiente, de tal manera que tanto los procesos del lenguaje, como los cognoscitivos, se desarrollan de manera simultánea conforme el niño transita a través de una serie de etapas previamente establecidas de desarrollo, que requieren interacciones más complejas con el entorno.

Además, Le Normand (Narbona, 2000 p. 3 I) manifiesta que desde esta perspectiva, el lenguaje "es examinado no solamente respecto a su organización en estructuras complejas, sino también en función de los contextos de los que depende parcialmente", permitiendo, de esta manera, explicar la precocidad y la rapidez del proceso de la adquisición del lenguaje y la plasticidad de los diferentes sistemas.

\section{DESLINDES CONCEPTUALES}

Antes de analizar los diferentes trastornos del lenguaje, es conveniente revisar una serie de conceptos con la finalidad de usar cada uno de ellos, en un mismo sentido, ya que los diferentes autores, dependiendo de su posición teórica y disciplina de la que procede; les han conferido connotaciones ligeramente diferentes, cuando no radicalmente opuestas, lo que origina confusión. 


\section{I Comunicación}

Esta referido al intercambio de información siendo un hecho social. Todos los animales se comunican, pero sólo los seres humanos disponen de un código tan complejo para comunicarse como es el lenguaje.

\subsection{Lenguaje}

Es el resultado de una actividad nerviosa compleja que permite la comunicación interindividual de estados psíquicos a través de la materialización de signos multimodales que simbolizan estos estados de acuerdo con una convención propia de una comunidad lingüística (Leocurus en Peña, 200 I , p. I).

\subsection{Componentes del lenguaje}

Acosta y Moreno (200 I) resumen una serie de conceptos con respecto a cada uno de los diferentes componentes.

- Pragmática: Es el estudio del lenguaje en contextos sociales, se interesa por las reglas que gobiernan el uso social del lenguaje en un contexto determinado.

- Fonología: Estudia los sonidos de la expresión lingüística de una lengua determinada desde un punto de vista funcional y abstracto. Ello se realiza a través de la organización de los sonidos en un sistema, utilizando sus caracteres articulatorios y la distribución de los contextos en que pueden aparecer.

- Fonética: Estudia el material sonoro, tratando de recoger la información más exhaustiva posible sobre la materia sonora bruta y sus propiedades, tanto fisiológicas como físicas, atendiendo a tres puntos de vista: producción (fonética articulatoria), transmisión (fonética acústica) y percepción (articulatoria auditiva).

- Semántica: Estudia el significado de los signos lingüísticos y sus posibles combinaciones en los diferentes niveles (palabras, frases, enunciados y discurso) de organización del sistema lingüístico

- Morfosintaxis: Componente lingüístico que se ocupa del estudio de las reglas que intervienen en la formación de las palabras y de las posibles combinaciones que estas puedan tener en el interior de las diferentes secuencias oracionales en las que se estructura una lengua.

\subsection{Habla}

Palomares (Sánchez, 1985, p. 1043), menciona que éste se refiere a la realización concreta, en un momento y lugar precisos, de un determinado código o lengua. 
Cada individuo selecciona, al hablar, los signos y reglas que la lengua pone a su disposición y que la persona extrae de su mente.

Por su parte, Saussure, citado también por Sánchez (1985), indica que es el funcionamiento de los signos del habla y de las relaciones encaminadas a la expresión del pensamiento individual; es decir, es la lengua en acción.

\subsection{Alteraciones del lenguaje}

Para Bashir (Puyuelo 2003, p. 102) es un grupo heterogéneo de desordenes en el desarrollo o adquisición del lenguaje, caracterizados principalmente por déficit en la comprensión, producción y uso del mismo. Algunas de estas alteraciones son crónicas y pueden persistir a lo largo de la vida, sin embargo, los síntomas, manifestaciones, efectos y severidad de los problemas cambian con el tiempo, como consecuencia del contexto y de las tareas de aprendizaje.

\subsection{Retraso del lenguaje}

Falta de desarrollo del lenguaje a la edad en que normalmente se presenta. Este concepto también se aplica a los niños que presentan patrones lingüísticos de niños de menor edad a la que realmente corresponde (Aguado, citado por Peña; 2002, $p, 229)$.

\subsection{Dificultades del lenguaje}

Concepto genérico y neutral referido a afectaciones del que permiten concebirlo en términos de necesidades educativas especiales, ya que la mayor parte de los problemas del lenguaje y de la comunicación se manifiestan, y en algunos casos se intensifican, en la situación de enseñanza - aprendizaje (Acosta; Moreno, 200 I, p. 4). Por lo tanto, la definición hace referencia a las afectaciones que sufre la persona en algún punto de la cadena de la comunicación (oído, cerebro u órganos periféricos del lenguaje); como en la interacción natural que se produce en distintos contextos de comunicación en los que cambian los interlocutores, los materiales y los eventos.

\subsection{Síndrome}

Conjunto de síntomas clínicos cuya aparición simultánea sugiere con elevada probabilidad un mecanismo patógeno o una localización común (Bruna; Suhevic citado por Junqué, 2003, p. 76).

\subsection{Signo}

En medicina un signo es una evidencia objetiva de la presencia de una enfermedad o desorden, opuesto a síntoma que es subjetivo (URL: http: // es.wikipedia.org). 


\section{10 Síntoma}

Sensación o cambio en el estado de salud de una persona. Un síntoma siempre es una sensación subjetiva, en contraposición a un signo que es un dato objetivo (URL: http: // es.wikipedia.org).

\section{CLASIFICACIÓN DE LOS TRASTORNOS DEL LENGUAJE EN EL NIÑO}

Abordar el tema de la clasificación de los trastornos de lenguaje, implica resaltar que, tanto la labor del especialista dedicado a la evaluación e intervención, como la del investigador, deben estar organizadas en base a un modelo que guié el análisis de la patología lingüística, aunque ello resulte complicado ya que a la fecha existe una amplia gama de alternativas para categorizar los diferentes trastornos, algunas de las cuales se presentarán a continuación. (Chevrie- Muller; Narbona, 200 I, p. 189).

\section{I Clasificación sobre la base de criterios neurolingüísticos}

La variedad de cuadros clínicos que se aprecia en esta clasificación, se debe a las siguientes razones:

a. La naturaleza modular del lenguaje, considera que cada cuadro clínico se encuentra en correspondencia a una deficiencia en alguno de los componentes lingüísticos, tanto a nivel comprensivo como expresivo.

b. Las asociaciones que se producen entre estas deficiencias.

c. Las patologías relacionadas con los defectos de la instrumentación de base (audición, aparato neuromotor especializado) y los déficit de los procesos intermediarios gnósicos y práxicos.

\subsection{Clasificación sobre la base de criterios etiológicos y psicopatológicos}

Para efectuar un correcto proceso de evaluación e intervención, es importante que se establezca con claridad, el criterio que se tendrá en cuenta para estimar una patología como congénita o adquirida, así, en esta disyuntiva se aprecia que los autores difieren en sus criterios de orden cronológico tal como se resume en el cuadro I elaborado en base a la información descrita por Chevrie- Muller y Narbona (200 I, p. 189). 


\section{Cuadro I: Criterios cronológicos para diferenciar entre patología del lenguaje congénita $o$ adquirida}

\begin{tabular}{|l|l|}
\hline Autor & Criterio \\
\hline Bates y cols. & $\begin{array}{l}\text { Agrupa sin distinción trastornos del lenguaje por } \\
\text { lesión cerebral desde los } 6 \text { meses a los } 6 \text { años. }\end{array}$ \\
\hline Van Hout & $\begin{array}{l}\text { Será patología adquirida desde fines del } 2 .^{\circ} \text { año, } \\
\text { cuando aparecen primeras manifestaciones de } \\
\text { sintaxis. }\end{array}$ \\
\hline Chevrie - Muller y Carbona & $\begin{array}{l}\text { Será adquirida a partir de los } 18 \text { a } 20 \text { meses, } \\
\text { con los primeros indicios de comunicación pre - } \\
\text { verbal. }\end{array}$ \\
\hline
\end{tabular}

Dioses; Quiroz, 2004

Sin embargo, aún con el avance actual de la ciencia y tecnología, es necesario indicar que la etiología y fisiopatología no son variables suficientes para apoyar una clasificación de los trastornos del lenguaje, pues todavía es muy difícil establecer una correlación significativa entre una sintomatología lingüística específica y una etiología y/o disfunción neurológica determinada; aunque también es importante reconocer, como lo señalan Chevrie - Muller y Narbona (200 I), que al momento ya se cuenta con datos más claros acerca de las bases genéticas de algunos problemas como los trastornos específicos de desarrollo del lenguaje oral, la dislexia y el tartamudeo.

\subsection{Clasificación propuesta por Narbona y Chevrie- Muller}

Estos autores plantean la necesidad de visualizar la actividad lingüística como el resultado del funcionamiento conjunto de los aspectos cognitivo y cerebral, de tal forma se evite caer en el reduccionismo al momento de efectuar una clasificación.

En este sentido, señalan que una clasificación satisfactoria debería considerar en forma simultánea, los criterios etiológico y lingüístico, proponiendo una categorización en un cuadro de doble entrada donde en las columnas se ubica el criterio etológico (congénito o adquirido) y en las filas, las patologías en los diferentes procesos y funciones del lenguaje, señalados en su modelo neuropsicolingüístico (Narbona; Chevrie - Muller, 200 I, p. 76) destinado a evaluar el lenguaje. 
a. Déficit en los instrumentos de base:

\begin{tabular}{|c|c|c|c|}
\hline \multicolumn{2}{|r|}{ Patologías } & \multicolumn{2}{|c|}{ ETIOLOGÍA } \\
\hline & & Congénito & Adquirido \\
\hline \multicolumn{2}{|c|}{ Déficit anatómicos } & $\begin{array}{l}\text { Disglosias por malformaciones } \\
\text { velopalatinas, labiales, linguales, } \\
\text { laringeas, maxilodentarias }\end{array}$ & \\
\hline & \multirow{2}{*}{$\begin{array}{l}\text { Déficit sensoriales } \\
\text { auditivos }\end{array}$} & \multicolumn{2}{|c|}{ Hipoacusias } \\
\hline & & $\begin{array}{l}\text { Déficit de transmisión } \\
\text { (malformaciones) } \\
\text { Déficit de percepción (genético, } \\
\text { tóxico, infeccioso) }\end{array}$ & $\begin{array}{l}\text { Déficit de transmisión (otitis) } \\
\text { Déficit de percepción } \\
\text { (infeccioso, tóxico) }\end{array}$ \\
\hline & $\begin{array}{l}\text { Trastornos del } \\
\text { orden y control } \\
\text { de la motricidad } \\
\text { faringobucal }\end{array}$ & $\begin{array}{l}\text { Disartrias y afemias } \\
\text { Parálisis labio-gloso-faríngeas } \\
\text { (síndrome pseudobulbar } \\
\text { aislado, o formando parte de } \\
\mathrm{PCl} \text { ) } \\
\text { Disartrias cerebelosas } \\
\text { Disartrias graves y afemias en } \\
\mathrm{PCl} \\
\text { Atetósica }\end{array}$ & $\begin{array}{l}\text { Lesiones neurológicas } \\
\text { periféricas o centrales: } \\
\text { traumáticas, vasculares } \\
\text { Enfermedades progresivas } \\
\text { (distonias secundarias) }\end{array}$ \\
\hline
\end{tabular}

b. Trastornos neurolingüísticos (gnoso-práxico-lingüísticos) incluye el lenguaje escrito:

\begin{tabular}{|c|c|c|c|}
\hline \multirow{2}{*}{\multicolumn{2}{|c|}{ Patologías }} & \multicolumn{2}{|c|}{ ETIOLOGÍA } \\
\hline & & Congénito & Adquirido \\
\hline \multirow{2}{*}{ 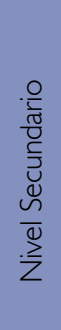 } & Déficit gnósicos & $\begin{array}{l}\text { Benignos: consecuencias en } \\
\text { articulación y ortografía. } \\
\text { Severos: agnosia verbal } \\
\text { congénita }\end{array}$ & $\begin{array}{l}\text { Síndrome de afasia- } \\
\text { epilepsia (Landau-Kleffner) }\end{array}$ \\
\hline & Déficit práxicos & $\begin{array}{l}\text { Benignos: trastornos de la } \\
\text { articulación: dislalias } \\
\text { Severos: dispraxia bucofacial }\end{array}$ & Apraxia bucofacial \\
\hline \multirow{2}{*}{ 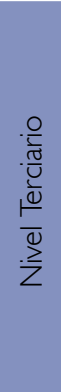 } & $\begin{array}{l}\text { Déficit lingüísticos } \\
\text { (com prensión - } \\
\text { expresión) }\end{array}$ & $\begin{array}{l}\text { Benignos: retraso simple de } \\
\text { palabra (fonología) o de lenguaje } \\
\text { (fonología, sintaxis, léxico) } \\
\text { Severos: disfasias (fonológicas, } \\
\text { sintácticas, disnómicas) }\end{array}$ & $\begin{array}{l}\text { Afasias por lesión } \\
\text { o disfunción uni o } \\
\text { bihemisférica (infecciones, } \\
\text { traumatismos, epilepsia) }\end{array}$ \\
\hline & $\begin{array}{l}\text { Déficit psicolingüísticos } \\
\text { Patología del lenguaje } \\
\text { escrito }\end{array}$ & $\begin{array}{l}\text { Trastorno semántico-pragmático } \\
\text { Dificultad de aprendizaje de } \\
\text { lectura y escritura: dislexia, } \\
\text { disortografía }\end{array}$ & $\begin{array}{l}\text { Pérdida de capacidades } \\
\text { anteriormente adquiridas: } \\
\text { Alexia, agrafía }\end{array}$ \\
\hline
\end{tabular}


c. Tartamudez

\begin{tabular}{|l|l|l|}
\hline \multirow{2}{*}{ Patologías } & \multicolumn{2}{|c|}{ ETIOLOGíA } \\
\cline { 2 - 3 } & \multicolumn{1}{|c|}{ Congénito } & \multicolumn{1}{c|}{ Adquirido } \\
\hline Tartamudeo & Tartamudeo (disfemia) & $\begin{array}{l}\text { Disfluencias (lesiones corticales } \\
\text { o subcorticales }\end{array}$ \\
\hline
\end{tabular}

d. Trastornos del lenguaje en psicopatología y carencias del entorno

\begin{tabular}{|l|l|l|}
\hline \multirow{2}{*}{ Patologías } & \multicolumn{1}{c|}{ ETIOLOGíA } \\
\cline { 2 - 3 } Estados deficitarios & \multicolumn{1}{|c|}{ Congénito } & \multicolumn{1}{c|}{ Adquirido } \\
\hline & $\begin{array}{l}\text { Deficiencia intelectual: } \\
\text { Homogénea. } \\
\text { Disarmónica (déficit verbal más } \\
\text { marcado que las capacidades } \\
\text { cognitivas no verbales). }\end{array}$ \\
\hline Trastornos de la comunicación & $\begin{array}{l}\text { Autismo infantil y su espectro. } \\
\text { Síndrome de Asperger }\end{array}$ & $\begin{array}{l}\text { Mutismo selectivo } \\
\text { Privaciones físicas, afectivas y } \\
\text { culturales }\end{array}$ \\
\hline
\end{tabular}

Tomado de Narbona (200 I,p. 191)

3.4 Clasificación de las alteraciones del lenguaje en función del componente del lenguaje alterado y de la etiología

Otra propuesta es la presentada por Puyuelo (Puyuelo; Rondal, 2003,p. I 04), quien señala que se podría realizar clasificaciones de los trastornos del lenguaje en función a las alteraciones de los componentes o la etiología.

a. En función al componente de lenguaje alterado:

- Problemas semánticos: Evidencia de una adquisición lenta de palabras y significados.

- Forma, fonología, morfología, sintaxis: Dificultad en establecerlas correspondencias correctas entre las formas de los niños y las de los adultos.

- Problemas morfológicos: Dificultad en el uso de formas gramaticales.

- Problemas sintácticos: Dificultad en la construcción de frases.

- Problemas pragmáticos: Problemas relacionados al uso social del lenguaje. 
- Déficit en la interacción entre los componentes del lenguaje: Alteración de las relaciones entre los diferentes componentes del lenguaje.

\section{b. En función a la etiología}

Este autor plantea que toda interferencia en la adquisición del lenguaje es denominada etiología, habiéndose identificado algunas de ellas tales como, limitaciones cognitivas, déficit sensoriales auditivos, problemas motores, relaciones sociales deficientes y falta de oportunidades lingüísticas en el entorno, pudiendo agruparse todas ellas en las siguientes categorías (Puyuelo; Rondal, 2003, p. 107):

- Factores centrales

Problemas de procesamiento central que incluyen alteraciones corticales, que inciden en aprendizaje a nivel cognitivo y lingüístico.

- Trastorno Especifico del Lenguaje: Existen déficit significativo en las habilidades del lenguaje que no está relacionadas a problemas cognitivos, motores, sensoriales.

- Retraso mental: La comprensión y producción del lenguaje dependerá del nivel cognitivo de cada individuo.

- Autismo: Existe alteración de la comunicación y en especial del componente pragmático.

- Déficit de atención e hiperactividad.

- Traumatismo craneoencefálico (TCE): En algunos casos puede afectar el lenguaje.

- Otros

- Factores periféricos

Que incluye los mecanismos sensoriales y motores.

- Problemas auditivos

- Problemas visuales

- Sordoceguera

- Problemas físicos 
- Factores del entorno y emocionales

Relacionados con los contextos de desarrollo o aspectos inherentes a la persona.

- Mixtos

Incluyen las alteraciones del lenguaje vinculadas a problemas cognitivos, sensoriales, motores.

Además de estas clasificaciones propuestas por diversos autores existen las propuestas en los manuales nosotáxicos internacionalmente admitidos.

\subsection{Clasificación de la Asociación Psiquiátrica Americana (1995)}

Trastornos de la comunicación.

(DSM - IV)

$(\mathrm{ClE}-10)$

315.31 Trastorno del lenguaje expresivo

F 80.।

315.31 Trastorno mixto del lenguaje receptivo-expresivo

F 80.2

315.39 Trastorno fonológico

F 80.0

307.0 Tartamudeo

F98. 5

307.9 Trastorno de la comunicación no especificado.

F 80.9

\subsection{Clasificación de la Organización Mundial de la Salud (1992)}

Los Trastornos del desarrollo psicológico (F80 - 89) tienen en común las siguientes características:

- Comienzo siempre en la primera o segunda infancia.

- Deterioro o retraso del desarrollo de las funciones que están íntimamente relacionadas con la maduración biológica del sistema nervioso central.

- Curso estable que no se ve afectado por las remisiones y recaídas que tienden a ser características de muchos trastornos mentales.

En la mayoría de los casos, las funciones afectadas son el lenguaje, el rendimiento de las funciones viso-espaciales o de coordinación de movimientos.

F80 Trastornos específicos del desarrollo del habla y del lenguaje. 
F80.0 Trastorno específico de la pronunciación.

F80.I Trastorno de la expresión del lenguaje.

F80.2 Trastorno de la comprensión del lenguaje.

F80.3 Afasia adquirida con epilepsia (síndrome de Landau-Kleffner).

F80.8 Otros trastornos del desarrollo del habla y del lenguaje.

F80.9 Trastorno del desarrollo del habla y del lenguaje sin especificación.

\subsection{Clasificación siguiendo el modelo de procesamiento de la información:}

Otra manera de clasificar los trastornos del lenguaje es siguiendo este modelo, de tal manera que pueden considerarse tres grandes grupos:

\section{a. Trastornos de entrada:}

Dentro de este grupo, se incluyen las alteraciones de la comunicación secundarias a déficit auditivo (hipoacusia, cofosis, presbiacusia, alteraciones en la discriminación auditiva, etc.) y disfunciones en la adquisición del lenguaje de las personas con déficit visual y alteraciones por deprivación bio-social.

\section{b. Trastornos de procesamiento:}

Se incluyen dentro de éste grupo los trastornos específicos del lenguaje, dificultades de la adquisición, trastornos adquiridos (afasias, agnosias, apraxias, dificultades en funciones ejecutivas, etc).

\section{c. Trastornos de salida:}

En éste grupo se involucra los trastornos del habla (disartria, anartria, disglosias, dislalias, etc.), trastornos del ritmo (disfemia, taquifemia, bradifemia, disprosodia), trastornos de la voz (disfonías orgánicas y funcionales), laringectomizados, deglución atípica, respiración bucal, disfagia, etc.

\section{SEMIOLOGÍA DE LOS TRASTORNOS DEL LENGUAJE EN EL NIÑO}

El conocimiento de la semiología de los trastornos del lenguaje permitirá realizar una evaluación precisa de las dificultades que pueda presentar el niño con relación a este proceso cognitivo complejo y a la vez modular, es así que en este apartado se describirán la sintomatología de una forma autónoma, sin importar el cuadro clínico en el que se encuentre. Se debe tener presente que esta sintomatología puede deberse a diversas causas, ya sean por déficit sensoriales o motores, cuadros patológicos mayores o una patología específica del lenguaje. 
De esta manera se procederá a realizar una descripción siguiendo el modelo neuropsicolingüístico de Chevrie - Muller y Narbona (200l), agrupándolos de acuerdo al nivel funcional del lenguaje.

\section{I Déficit Gnósicos (Nivel 2 MNPL)}

La posibilidad de interpretar la información sonora, y en especial del lenguaje oral, depende no sólo de la audición, condición biológica indispensable (poder hacer), sino también del aprendizaje (saber hacer). El déficit gnósico en este nivel, denominada agnosia auditiva se caracteriza por la incapacidad para reconocer sonidos y ruidos de la vida cotidiana a pesar de percibirlos correctamente; siendo clínicamente muy rara (Barbizet; Duizabo; 195, p. 64).

Cuando el problema se centra en la incapacidad para el reconocimiento del lenguaje oral se denomina agnosia verbal, en este último caso es probable que el sujeto si sea capaz de reconocer ruidos no verbales.

En el cuadro 2 se describe el proceso requerido para que el niño logre el reconocimiento auditivo y las dificultades que se presentan en el caso de las agnosias señaladas.

\section{Cuadro 2: Agnosia auditiva y agnosia verbal}

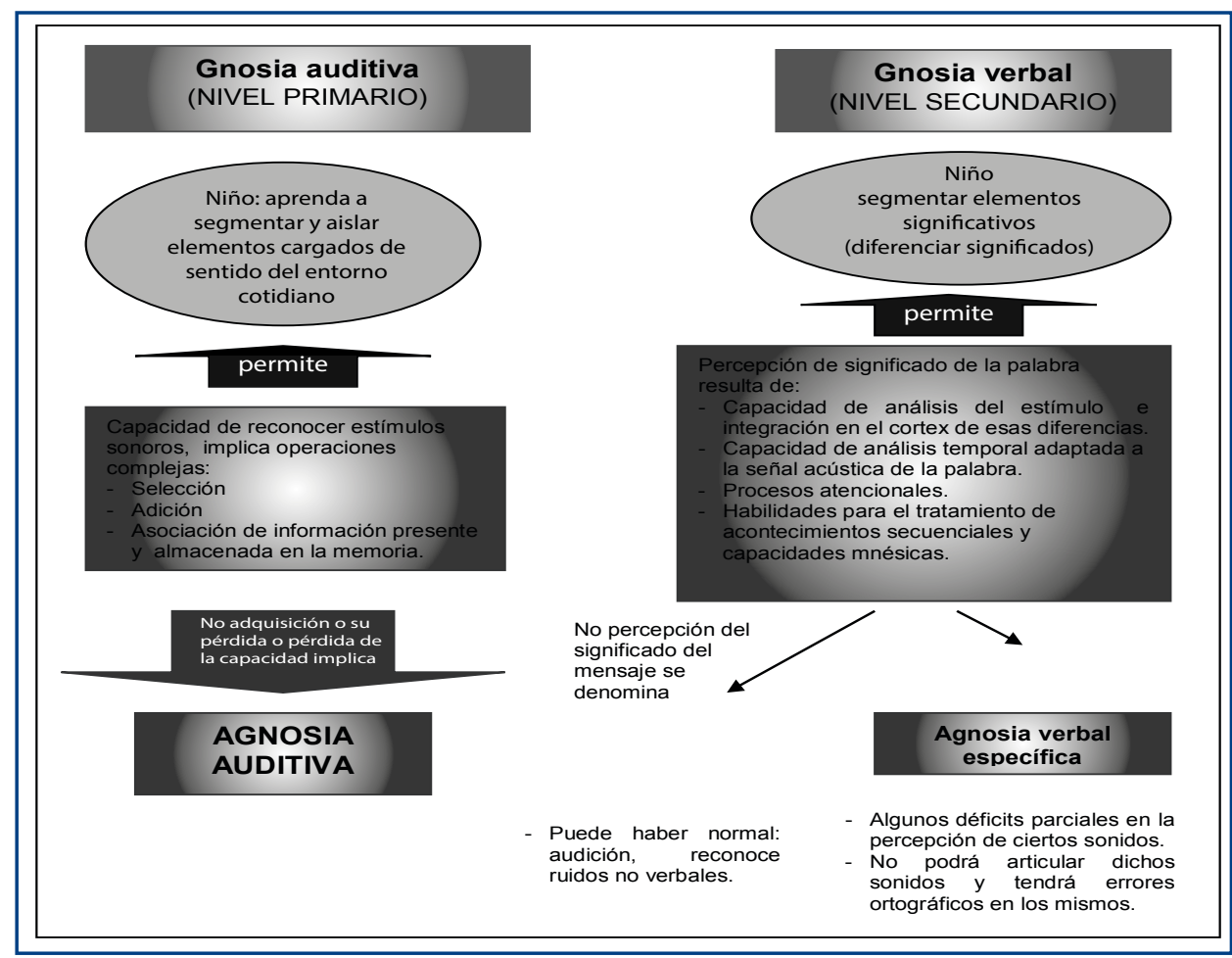




\subsection{Trastornos de la comprensión (Nivel 3 del MNPL)}

Los problemas en la comprensión del lenguaje están referidos a la imposibilidad de entender los diferentes marcadores morfológicos (tiempo, género, número), estructuras sintácticas, sobre todo de preguntas abiertas, o comprender enunciados complejos.

Estos déficits a nivel terciario o lingüístico generalmente son detectados mediante una exploración con pruebas específicas que evalúen el vocabulario pasivo (nivel 3 - c del MNPL), la morfosintaxis (nivel 3- b del MNPL) y la interpretación de enunciados y relatos (nivel 3 - d del MNPL).

El estudio de la comprensión verbal implica analizar también el nivel cognitivo del niño ya que este jugará un papel importante al permitir permite la asociación del mensaje recibido con los conocimientos previos que tenga.

\subsection{Déficit práxicos. Trastornos de la articulación (nivel 5 del MNPL)}

En los déficits práxicos, denominados en el niño dispraxias, existe la dificultad o imposibilidad de ejecutar movimientos (Azcoaga, 1993, p. 78) del área bucal, sean estos simples o complejos, en ausencia de un trastorno motor que impida realizar los movimientos. De opinión semejante son Junqué; Bruna y Mataró (2004, p. 73) quienes la definen como una alteración en la ejecución de determinados movimientos aprendidos en respuesta a una orden y fuera de contexto, que no son el resultado de problemas sensoriales o motores, falta de coordinación o deterioro intelectual.

En esta perspectiva las praxias del área bucal pueden ser de dos tipos. Unas se organizan a través de movimientos más o menos complejos relacionándose con las funciones de deglución, soplo, producción de ruidos o "clics imitativos". Otras, las praxias articulatorias o fonéticas, son indispensables para la realización del lenguaje oral.

Las praxias fonéticas se aprenden progresivamente, a través del control y coordinación fina de grupos musculares, donde la complejidad de los movimientos articulatorios determina su orden de aparición. Estas praxias implican un proceso, que se inicia con el uso de las capacidades neuromotrices básicas del aparato fonatorio, el reconocimiento del fonema del lenguaje, y por último, la intención de reproducirlo. 
Cuadro 3: Trastornos articulatorios o dislalias

\section{TRASTORNOS DE ARTICULACIÓN O DISLALIAS}

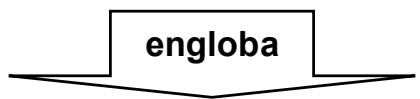

- Retraso de la adquisición de uno o varios rasgos articulatorios (en general aparece entre los 4, 5 ○ 6 años )

- Alteración de los rasgos articulatorios, rotacismo velar, rotacismo interdental, omisiones (persisten en la edad adulta sino son tratados).

Quiroz, 2004

Por otro lado, Chevrie- Muller (Narbona 200 I, p. 197) se refiere también a la apraxia verbal grave, aunque indica que su existencia está en duda porque se cree muy difícilmente que su origen se deba sólo a una limitada producción de movimientos articulatorios, pensándose mas bien está ligada a un trastorno en la secuencia de fonemas.

\subsection{Trastornos fonológicos o trastornos del habla (Nivel 4 - D Del MNPL)}

Al analizarse el lenguaje, hay que tener en cuenta que el nivel fonológico se refiere a la organización de sonidos en palabras; mientras que el nivel fonético a la realización material de los sonidos del lenguaje

En el nivel fonológico, la programación (elección) y la secuenciación de los sonidos que forman parte de una palabra, pueden estar perturbadas por una serie de dificultades como elisiones u omisiones, adiciones, sustituciones, metátesis u inversiones y asimilaciones.

Diversos autores (Narbona, Chevrie - Muller, 200 I; Torres, 1996; Puyuelo y Rondal, 2003; Acosta y Moreno, 200 I) indican que las alteraciones son "normales" a cierta edad, pero su persistencia si es considerada patológica.

Cuadro 4: Características de las alteraciones fonéticas y fonológicas

\begin{tabular}{|l|l|}
\multicolumn{1}{|c|}{ ALTERACIONES FONÉTICAS } & \multicolumn{1}{|c|}{ ALTERACIONES FONOLÓGICAS } \\
\hline Las alteraciones de fonemas son sistemáticas & $\begin{array}{l}\text { Las alteraciones de fonemas NO son } \\
\text { sistemáticas }\end{array}$ \\
\hline $\begin{array}{l}\text { Los errores se presentan en sonido aislado, } \\
\text { sílaba y palabra }\end{array}$ & $\begin{array}{l}\text { Los fonemas alterados en palabras se repiten } \\
\text { correctamente de manera aislada y en sílabas }\end{array}$ \\
\hline Los errores son constantes & $\begin{array}{l}\text { Las dificultades aumentan con la longitud de la } \\
\text { palabra }\end{array}$ \\
\hline El tipo de error es el mismo & $\begin{array}{l}\text { Una misma palabra se altera de manera } \\
\text { diferente cada vez }\end{array}$ \\
\hline
\end{tabular}




\subsection{Apraxia del habla}

El estudio de las patologías del desarrollo del lenguaje, tanto a nivel fonético como fonológico no está bien diferenciado, ya que ambas dificultades suelen coexistir a menudo.

Chevrie - Muller (Narbona; Chevrie - Muller, 200I, p. 198) indica que Aram y Nation, diferencian claramente los niveles fonético y fonológico, no obstante resaltan que en las apraxias del habla coexisten dificultades de ambos componentes del lenguaje, concluyendo además, que según la edad, alguno de los dos componentes predomina, así en los niños pequeños, predominan notablemente los problemas fonéticos asociado a un repertorio articulatorio restringido; mientras que en los niños más grandes, los principales problemas son a nivel fonológico, presentando alteraciones en la colocación de fonemas, secuenciación de sílabas y alteraciones prosódicas.

Cabe señalar que la sintomatología en este nivel se observa desde los niveles moderados hasta la ausencia del lenguaje oral (apraxias severas).

Complementariamente, Shriberg y cols. (Narbona; Chevrie - Muller, 200 I, p. 198) señalan la existencia de un trastorno motor del habla, debido a déficit de secuenciación de los fonemas en la fase pre -articulatoria, el mismo que podría persistir hasta alrededor de los ocho años.

\subsection{Trastornos de la prosodia (Niveles 3 y 4 de recepción y expresión del MNLP)}

La prosodia se ubica en el tercer nivel de funcionamiento del MNLP de Chevrie Muller y Narbona, e incluye fenómenos como la entonación, la acentuación, la fluidez y el ritmo del habla. La mayor dificultad dentro de este nivel es la disfluencia, sin embargo, se debe considerar el análisis de las dificultades de este aspecto en todas las patologías del lenguaje.

Narbona y Chevrie - Muller (200 I, p. 198) indican que Chalumeau destaca la importancia que tiene el análisis prosódico en el diagnóstico, pronóstico y terapia del TEDL.

\subsection{Trastornos morfosintácticos de expresión (4-C MNPL)}

Esta dificultad se refiere a las limitaciones que tiene el individuo para asociar palabras en frases y a los problemas que tiene para manejar adecuadamente los diferentes componentes de la gramática.

Los trastornos en este nivel son de una severidad variable, y van desde una torpeza sintáctica a un agramatismo caracterizado por omisiones de segmentos funcionales 
(artículos, pronombres), ausencia de conjugación del verbo y alteraciones en el orden de los componentes de la frase.

\subsection{Disnomias. Trastornos de la recuperación de las palabras (nivel 4-b del MNPL)}

La presencia de estos trastornos se puede dar tanto en el niño, como en el adulto. Este trastorno ha sido poco estudiado debido a su sutilidad ya que puede ser detectado sólo a través de instrumentos específicos.

\section{Cuadro 5: Disnomia en niños y adultos}

\begin{tabular}{|c|c|}
\hline $\begin{array}{l}\text { NIÑO : Anomias en el desarrollo o Disnomia } \\
\text { (Denckla) }\end{array}$ & $\begin{array}{l}\text { ADULTO : Anomias adquiridas } \\
\text { (Le Dorze y Nespoulous) }\end{array}$ \\
\hline $\begin{array}{l}\text { - La disnomia puede presentarse de } \\
\text { forma aislada o asociada a trastornos del } \\
\text { lenguaje } \\
\text { - Alteración en la fluidez del discurso, con } \\
\text { presencia de titubeos o repeticiones. } \\
\text { - Alteración en la recuperación de palabras. } \\
\text { - La evaluación se realiza a través de } \\
\text { pruebas de recuperación fuera de } \\
\text { contexto. }\end{array}$ & $\begin{array}{l}\text { - Parte de otro cuadro clínico o puede ser } \\
\text { - } \text { Puro. } \\
\text { semántición de la representación: a nivel } \\
\text { - Dificultad de la representación a partir } \\
\text { de las características fonológicas del } \\
\text { léxico. }\end{array}$ \\
\hline
\end{tabular}

Quiroz, 2004

\subsection{Trastornos semántico - pragmáticos (Nivel 3d - 4a del MNPL)}

La alteración en el uso funcional del lenguaje puede ser de gravedad variable; su detección sólo es factible a través de una observación minuciosa y con instrumentos específicos para la evaluación de dichos componentes.

\section{Cuadro 6: Síndrome semántico - pragmático (Rapin y Allen)}

\begin{tabular}{l|l}
\multicolumn{1}{|c|}{ Características positivas } & \multicolumn{1}{c}{ Alteraciones } \\
\hline - Habla fluida. & - No hay comprensión de la función y utilidad \\
- Frases correctamente estructuras. & del lenguaje, tanto para los intercambios \\
- Vocabulario adecuado para su edad. & interpersonales como para ellos mismos. \\
& - Realizan preguntas, pero no atienden a las \\
& respuestas. \\
- Comprensión literal \\
- Se presentan en los niños autistas, así como en \\
los niños con síndrome de asperger y niños con \\
hidrocefalia.
\end{tabular}




\section{Referencias bibliográficas}

Asociación Psiquiátrica Americana (1995) Manual diagnóstico y estadístico de los trastornos mentales DSM - IV. Barcelona: Ed. Masson

Acosta; M. (200 I). Dificultades del lenguaje en ambientes educativos: Del retraso al trastorno específico del lenguaje. Barcelona: Ed. Masson

Azcoaga y colb. ( 1983). Las funciones cerebrales superiores y sus alteraciones en el niño y en el adulto (neuropsicología). Buenos Aires. Paidós

Barbizet, D (1985). Manual de Neuropsicología. Edit. Masson. Barcelona: Ed. Masson

Barrera, F. (1988) Adquisición y desarrollo del español: introducción para un recuento bibliográfico. Caracas: IUPC, CILLAB

Chomsky, N. (1975). Syntactic structures. Mounton. La Haya.

Greene, J. (1980). Psicolingüística: Chomsky y la piscología. México: Ed. Trillas

Junqué B. (2004). Neuropsicología del lenguaje: Funcionamiento normal y patológico. Rehabilitación. Barcelona: Ed. Masson

Lenneberg, E. (1975). Fundamentos biológicos del lenguaje. Madrid: Ed. Alianza

Mc. Neill, D. (1970). The acquision of language: the study of devolpmental psycolinguistics. Harper and Row. NY.

Narbona,CH. (200 I). El lenguaje del niño. Desarrollo normal, evaluación y trastornos. Barcelona: Ed. Masson

Organización Mundial de la Salud (1992). Clasificación internacional de enfermedades. Madrid: WHO y Meditor

Peña, J. (1994). Manual de Logopedia. Barcelona: Ed. Masson

Piaget, J. ( 1976). El lenguaje y el pensamiento en el niño. Buenos Aires: Ed. Guadalupe

Puyuelo; R. (2003). Manual de desarrollo y alteraciones del lenguaje. Barcelona: Ed. Masson

Toorres, J. ( 1996). Cómo detectar y tratar las dificultades en el lenguaje oral. Barcelona:

Ed. CEAC

Recuperado de: URL: http: // es.wikipedia.org 